\title{
Ecological Study on Differences in COVID-19 Fatality among Wuhan, Rest of Hubei, and Rest of China
}

\author{
Youfu $\mathrm{Ke}^{1, *}, \mathbb{D}$, Jianli Cui ${ }^{2}, \mathbb{D}$, Yunkeung Wong ${ }^{3,}$ (D) \\ ${ }^{1}$ Clinical Division, School of Chinese Medicine, Hong Kong Baptist University, Hong Kong 999077, China \\ ${ }^{2}$ School of Health and Biomedical Sciences, RMIT University, Melbourne, Victoria 3083, Australia \\ ${ }^{3}$ Hong Kong Institute of Vocational Education, Hong Kong, 999077, China
}

\section{ARTICLE INFO}

\section{Article History}

Received 21 April 2020

Accepted 22 August 2020

\section{Keywords}

SARS-CoV-2

COVID-19

ecological study

cross-sectional study

epidemiological investigation

\begin{abstract}
Background: China's vigorous anti-COVID-19 campaign has been going on for three months since January 20, which has contained the spread of the virus across China.

Objectives: Epidemiological investigations found that COVID-19 fatality rates in Wuhan, rest parts of Hubei province except Wuhan (Rest of Hubei) and rest parts of Mainland China except Hubei province (Rest of China) were different. An ecological study was conducted to analyze the reasons and provide the world with China's anti-COVID-19 epidemic experiences.

Methods: Infected cases from Mainland China were divided into three populations: Wuhan, Rest of Hubei and Rest of China. Methods were based on The Novel Coronavirus Infected Pneumonia Diagnosis and Treatment Standards. Total confirmed cases, daily severe cases, total deaths from February 12 to April 20 were collected for statistical analysis.

Results: 50,333 total confirmed cases in Wuhan made up the most substantial part by comparison with 17,795 in Rest of Hubei and 14,630 in Rest of China, respectively. In the early stage of the COVID-19 epidemic, daily severe cases in Wuhan accounted for the majority, and as the epidemic controlled, severe cases in all three populations decreased. Total deaths in Wuhan constituted the most significant proportion, with the highest 3869 in contrast to 643 in Rest of Hubei and 120 in Rest of China. The fatality rates in Wuhan ranged from $2.82 \%$ to $7.69 \%$, much higher than $1.80-3.61 \%$ in Rest of Hubei, and $0.49-0.88 \%$ in Rest of China. Pearson chi-square test for fatality rates in the three populations demonstrated significant differences $(p<0.01)$.

Conclusion: The ecological comparison study among the three populations have proved that social distancing, quarantine, lockdown, cutting off sources of infection and transmission routes, early detection, early isolation, early treatment are all vital to control the epidemic by reducing COVID-19 confirmed cases, severe cases and the fatality rate.
\end{abstract}

(C) 2020 The Authors. Published by Atlantis Press International B.V.

This is an open access article distributed under the CC BY-NC 4.0 license (http://creativecommons.org/licenses/by-nc/4.0/).

\section{INTRODUCTION}

China's vigorous anti-COVID-19 campaign has adopted ancient methods for prevention and control of infectious diseases [1] and has been going on for 3 months since January 20. Despite the concealment and delay at the beginning [2], it seems that the epidemic situation in China has been controlled, but it has also evolved into a pandemic, which has been breaking out in more than 200 countries. China provided the world with a month of preparation time and a wealth of experience in fighting the COVID-19 epidemic.

\section{OBJECTIVES}

Early epidemiological cross-sectional observations showed that COVID-19 fatality rates in Wuhan, Rest of Hubei and Rest of China

\footnotetext{
"Corresponding author.Email: keyoufu@gmail.com

Data availability statement: The data that support the findings of this study are openly available in the Health Commission of Hubei province at http://wjw.hubei.gov.cn/fbjd/ dtyw/, and the National Health Commission of the People's Republic of China at http:// www.nhc.gov.cn/xcs/yqtb/list_gzbd.shtml.
}

were different. However, China-WHO Joint Expert Expedition Group on Novel Coronavirus Pneumonia announced at a press conference in Wuhan on February 25 that the whole gene sequencing of 104 Novel Coronavirus strains isolated in different locations confirmed that the homology reached $99.9 \%$, indicating that the virulence could not be used to explain differences in fatality rates. An ecological comparison study is an epidemiological research method, which observes the differences in the distribution of a disease in different populations or regions; or compares the incidence and mortality of diseases in populations with different levels of exposure to provide clues to the etiology. Therefore, ecological studies should be used when large-scale comparisons are needed to study the population-level effect of exposures on a disease condition [3].

We had observed the population-level differences in the distribution of COVID-19 such as total confirmed cases, daily severe cases, total deaths, and fatality rates among Wuhan, Rest of Hubei and Rest of China to analyze and put forward the reasons to answer this urgent question, the conclusions were as follows. 


\section{METHODS}

\subsection{Study Population}

The study populations were the confirmed COVID-19 cases in Mainland China. Confirmed cases from Mainland China were divided into three groups: Wuhan, Rest of Hubei and Rest of China. Prevention, quarantine, and treatment were based on The Novel Coronavirus Infected Pneumonia Diagnosis and Treatment Standards [4]. The criteria for confirmed cases, severe cases, differential diagnoses, and clinical classification were adopted from the guidelines [5].

\subsection{Data Collection}

The data collected were based on those released daily by the Health Commission of Hubei province and the National Health Commission of the People's Republic of China on China's fight against the COVID-19 epidemic. The data included total confirmed cases, daily severe cases, total deaths from February 12 to April 20. The daily number represents the number of cases on a particular day; the total number represents the cumulative number of infected cases.

\subsection{Data Analysis}

The data were input into IBM SPSS Statistics 25 Product Version (IBM Corporation, New York) for data processing using statistical analyses such as the Pearson chi-square test to process the count data. ArcGIS Online was applied to draw a statistical map. Microsoft Office 2019 Excel was applied for drawing figures.

\subsection{Ethical Considerations}

The data used in this article were extracted from China's fight against COVID-19 epidemic data, which were available to the general public. No patients were directly involved in this study. Therefore, ethical approval was not required, and patient consent for publication is not required either.

\section{RESULTS}

As of April 20, the total confirmed cases, total deaths, and fatality rates of Wuhan, Rest of Hubei, Rest of China, were summarized in a statistical map (Figure 1).

50,333 total confirmed cases in Wuhan made up most substantial part by comparison with 17,795 in Rest of Hubei and 14,630 in Rest of China respectively (Figure 2).

In the early stage of the COVID-19 epidemic, daily severe cases in Wuhan accounted for the majority, and as the epidemic controlled, severe cases in all three populations decreased (Figure 3).

Total deaths in Wuhan constituted the most significant proportion, with the highest 3869 in contrast to 643 in Rest of Hubei and 120 in Rest of China (Figure 4).

The fatality rates in Wuhan ranged from $2.82 \%$ to $7.69 \%$, much higher than $1.80-3.61 \%$ in Rest of Hubei, and $0.49-0.88 \%$ in Rest of China. The average fatality rate in Mainland China

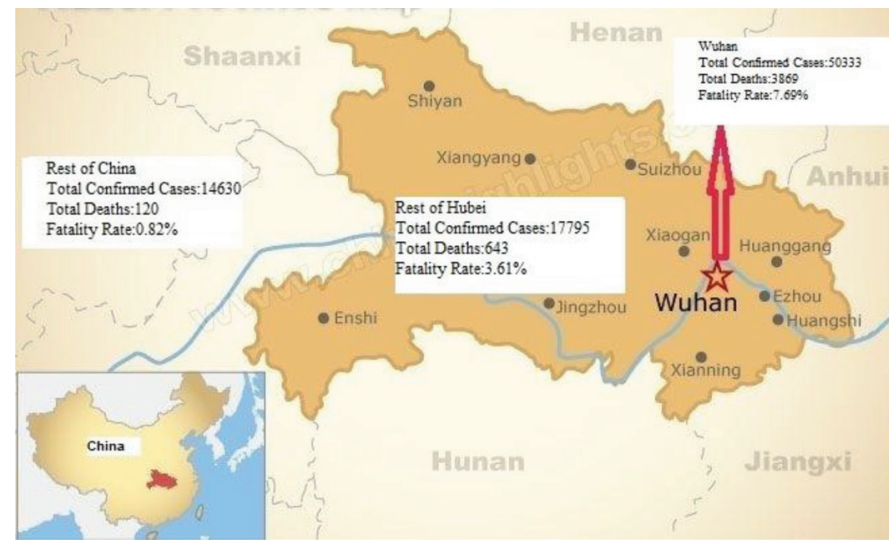

Figure 1 Total confirmed cases, total deaths and fatality rates in Wuhan, Rest of Hubei, Rest of China on April 20, 2020.

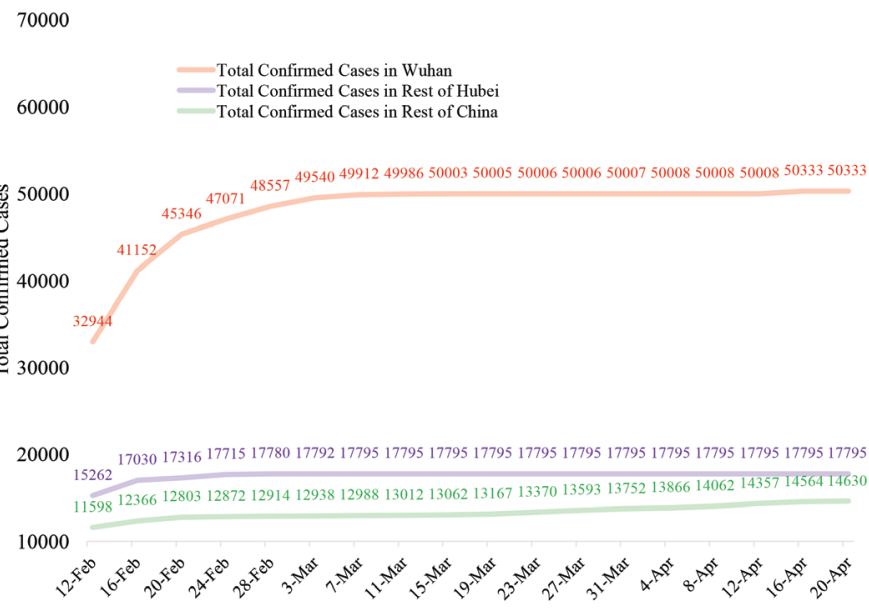

Figure 2 Total confirmed cases in Wuhan, Rest of Hubei, Rest of China.

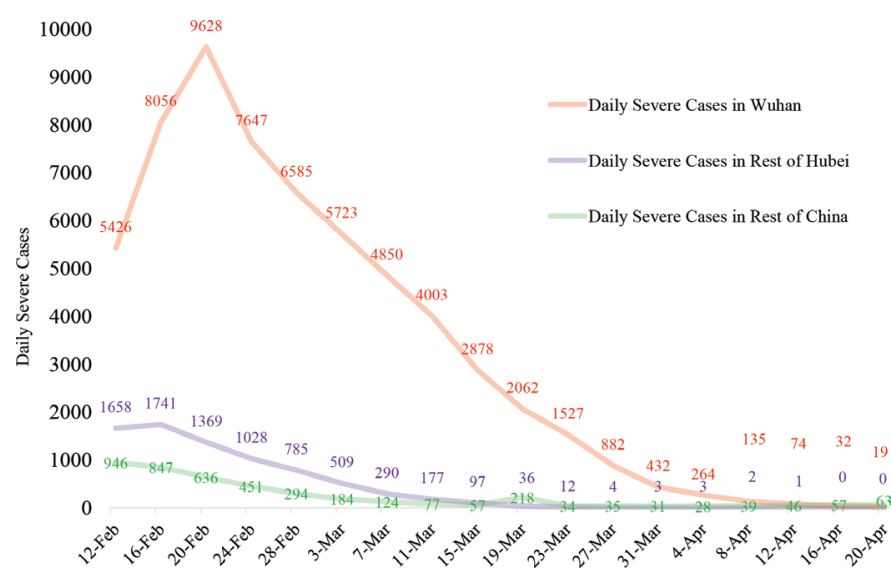

Figure 3 Daily severe cases in Wuhan, Rest of Hubei, Rest of China.

was $2.16-5.60 \%$. Pearson chi-square test for fatality rates in the three populations demonstrated significant differences $(p<0.01)$ (Figure 5).

Because medical staff in Wuhan was busy rescuing patients as well as lack of nucleic acid detection and the ability to treat patients in the early stage of the epidemic, and the public health statistics system was incomplete, resulting in late registration, 


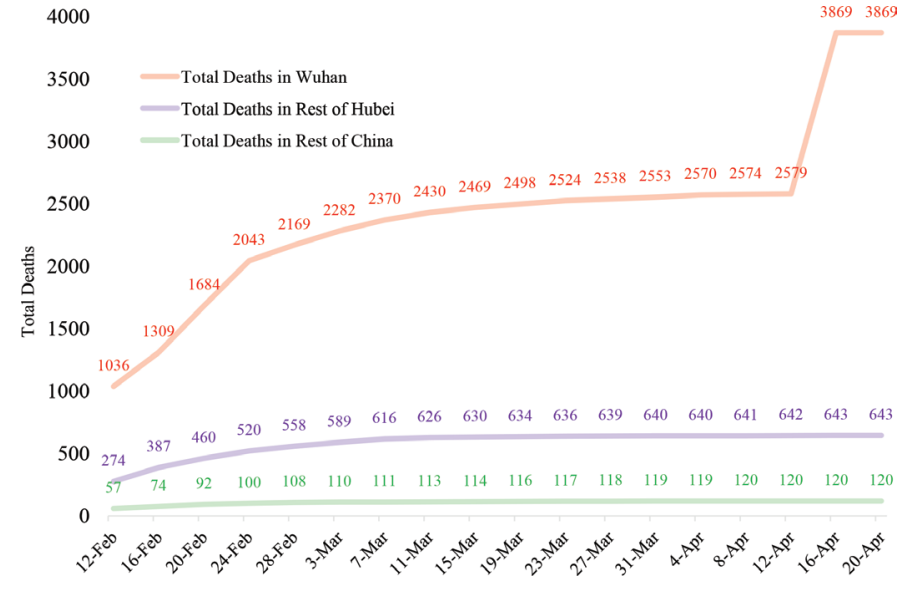

Figure 4 Total deaths in Wuhan, Rest of Hubei, Rest of China.

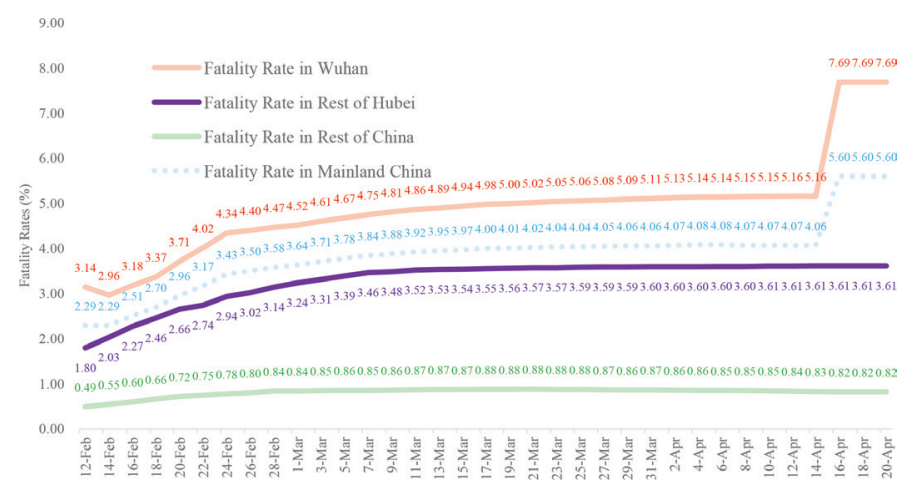

Figure 5 Fatality rates in Wuhan, Rest of Hubei, Rest of China, Mainland China.

missed registration, misregistration of some cases. At 11:00 on April 17, Wuhan City, Hubei Province issued the "Notice on the Correction of the Confirmed Cases and Deaths of COVID-19 in Wuhan", which states that as of 24:00 on April 16, a total of 50,333 confirmed cases (an increase of 325), 3869 deaths (an increase of 1290), and 4635 discharged cases (a decrease of 965) were registered in Wuhan. That was the reason why the data and graphics in Figure 4, five on April 16 had changed a lot.

\section{DISCUSSION}

Wuhan is the birthplace of COVID-19 epidemic, other cities and counties in Hubei province are the closest to Wuhan, therefore are the easiest to be affected. Before the Spring Festival, more than 5 million people had flowed out of Wuhan. After lockdown on January 23, the epidemic was restricted to Wuhan [2]. This is why total confirmed cases in Wuhan made up most substantial part, 50,333 by comparison with 17,795 in Rest of Hubei and 14,630 in Rest of China respectively. Moreover, from the ecological comparison of the three populations, it was found that the closer to Wuhan, the worse the epidemic; the more confirmed cases; the higher the proportion of severe cases; the higher the fatality rates. The COVID-19 epidemic was the most serious in Wuhan, less severe in Rest of Hubei, the least serious in Rest of China. In the early stage of the COVID-19 epidemic, daily severe cases in Wuhan accounted for the majority. Total deaths in Wuhan constituted the most significant proportion, with the highest 3869 in contrast to 643 in Rest of Hubei and 120 in Rest of China. The fatality rates in Wuhan ranged from $2.82 \%$ to $7.69 \%$, much higher than $0.49-0.88 \%$ in Rest of China, and $1.80-3.61 \%$ in Rest of Hubei. Pearson chi-square test for fatality rates in the three populations demonstrated significant differences $(p<0.01)$.

China-WHO Joint Expert Expedition Group on Novel Coronavirus Pneumonia announced at a press conference in Wuhan on February 25 that the whole gene sequencing of 104 Novel Coronavirus strains isolated in different locations confirmed that the homology reached $99.9 \%$, indicating that the virulence could not be used to explain differences in fatality rates. Consequently, it makes sense to deduce that the differences in the population-level effect of exposures on COVID-19 epidemic have caused differences in the distribution of confirmed cases, the proportion of severe cases, and the fatality rates. When COVID-19 occurred, fever clinics in Rest of Hubei, especially in Wuhan, were overcrowded with fever patients, causing severe cross-infection, coupled with the lack of beds in hospitals; doctors could only let them go home and isolate themselves, thus further resulting in the numbers of infected and severe patients. The fact that Wuhan had to quickly build multiple hospitals and square cabin hospitals to accommodate COVID-19 patients gave a perfect answer.

A study by the University of Hong Kong pointed out that the $R_{0}$ for SARS-CoV-2 was 2.68. The epidemic doubling time was 6.4 days [6]. Wuhan is the major transportation hub of central China. The outbreak in major cities in China and overseas with close transport links to Wuhan could become inevitable unless public health interventions are implemented immediately. China has implemented city-wide quarantine of Wuhan and nearby cities since January 23, 2020. Community-wide social distancing measures and other non-pharmaceutical interventions such as face masks and improved personal hygiene might be able to reduce influenza transmission by up to $50 \%$ as previous studies demonstrated [7]. Mitigation measures that limit population mobility to reduce within-population contact rates through the cancellation of mass gatherings, school closures, work-fromhome arrangements, wearing masks, and temperature screening measures in airports and train stations have prevented the spread of the epidemic in China.

The COVID-19 epidemic in the three populations in China has proved that the better the prevention and treatment are, the less the price will be paid, and the less the fatality rate will be. China provided the world with a month of preparation time $[8,9]$ and a wealth of experiences in fighting the epidemic [10,11]: the delay in social distancing, quarantine, lockdown, cutting off sources of infection and transmission routes, early detection, early isolation, early treatment could lead to an increase in a fatality. Because the critical period of prevention and control was delayed in Wuhan due to some reason [2] and the fact that the clinical manifestations and diagnosis of the disease were not well understood at first [12]. Therefore, the fatality rate in Wuhan was $7.69 \%$, higher than $3.61 \%$ in Rest of Hubei, much higher than $0.82 \%$ in Rest of China.

China's anti-COVID-19 epidemic experiences have been proved to be vital to control the epidemic by reducing COVID-19 confirmed cases, severe cases and the fatality rate [13-15]. The United States, Spain, Italy, France, Germany, and United Kingdom 
are the six countries with much smaller populations than China, and geographically farther away from the epidemic centre Wuhan than Rest of Hubei and Rest of China. They should have been less affected if appropriate anti-epidemic measures had been taken. There may be several reasons for these countries having more cases and deaths, but all the six countries share a common feature: reluctance to wear masks and maintain social distancing at the beginning, which may result in more confirmed cases and deaths than China, confirming the importance of China's anti-COVID-19 epidemic experiences.

This study is an ecological comparison study of epidemiological data in Wuhan, Rest of Hubei, and Rest of China. COVID-19 originated in Wuhan, Rest of Hubei and Rest of China are more accessible to be affected than other parts of the world at the early stage of the outbreak because of the similar geographical distribution of the population. Therefore, China's anti-COVID-19 epidemic experiences have vital guiding significance for the world's antiCOVID-19 pandemic at a late stage.

The data used in this study only provided total confirmed cases, daily severe cases, and total deaths. Other specific information about gender, age, symptom statistics, days of hospitalization, medication status, primary disease status etc. were not provided. Conclusions drawn were only based on the epidemic situations in Wuhan, Rest of Hubei and Rest of China. Many reasons are leading to the higher fatality rates in Wuhan than in Rest of Hubei and Rest of China, such as the false-negative rate, and the lower number of people diagnosed than the actual number of infections, which is not possible to elaborate here, in general, the effects of the three populations are similar.

\section{CONCLUSION}

The ecological comparison study among the three populations has proved that social distancing, quarantine, lockdown, cutting off sources of infection and transmission routes, early detection, early isolation, early treatment are all vital to control the epidemic by reducing COVID-19 confirmed cases, severe cases and the fatality rate.

\section{CONFLICTS OF INTEREST}

The authors declare they have no conflicts of interest.

\section{AUTHORS' CONTRIBUTION}

YK participated in conceptualization, data collection, statistical analysis, manuscript drafting, and approval of the final version of the manuscript. JC participated in database management, statistical analysis, critical review of the manuscript, and approval of the final version of the manuscript. YW participated in database management, manuscript revision, and approval of the final version of the manuscript.

\section{FUNDING/SUPPORT}

This research is self-funded.

\section{REFERENCES}

[1] Wilder-Smith A, Freedman DO. Isolation, quarantine, social distancing and community containment: pivotal role for old-style public health measures in the novel coronavirus $(2019-\mathrm{nCoV})$ outbreak. J Travel Med 2020;27;taaa020.

[2] Wang FS, Zhang C. What to do next to control the 2019-nCoV epidemic? Lancet 2020;395;391-3.

[3] Munnangi S, Boktor SW. Epidemiology of study design. Treasure Island (FL): StatPearls Publishing; 2020.

[4] The novel coronavirus infected pneumonia diagnosis and treatment standards (the sixth trial edition). Beijing: National Health Commission of the People's Republic of China and National Administration of Traditional Chinese Medicine of the People's Republic of China; 2020. Available from: http://www.nhc.gov. cn/yzygj/s7653p/202002/8334a8326dd94d329df351d7da8aefc2. shtml (accessed April 21, 2020).

[5] Jin YH, Cai L, Cheng ZS, Cheng H, Deng T, Fan YP, et al. A rapid advice guideline for the diagnosis and treatment of 2019 novel coronavirus (2019-nCoV) infected pneumonia (standard version). Mil Med Res 2020;7;4.

[6] Wu JT, Leung K, Leung GM. Nowcasting and forecasting the potential domestic and international spread of the 2019-nCoV outbreak originating in Wuhan, China: a modelling study. Lancet 2020;395;689-97.

[7] Bootsma MCJ, Ferguson NM. The effect of public health measures on the 1918 influenza pandemic in U.S. cities. Proc Natl Acad Sci U S A 2007;104;7588-93.

[8] Koonin LM. Novel coronavirus disease (COVID-19) outbreak: now is the time to refresh pandemic plans. J Bus Contin Emer Plan 2020;13;1-15.

[9] Akin L, Gözel MG. Understanding dynamics of pandemics. Turk J Med Sci 2020;50;515-19.

[10] Wang YY, Jin YH, Ren XQ, Li YR, Zhang XC, Zeng XT, et al. Updating the diagnostic criteria of COVID-19 "suspected case" and "confirmed case" is necessary. Mil Med Res 2020;7;17.

[11] Ding Z, Xie L, Guan A, Huang D, Mao Z, Liang X. Global COVID-19: warnings and suggestions based on experience of China. J Glob Health 2020;10;011005.

[12] Lu H, Stratton CW, Tang YW. The Wuhan SARS-CoV-2-What's next for China. J Med Virol 2020;92;546-7.

[13] Hellewell J, Abbott S, Gimma A, Bosse NI, Jarvis CI, Russell TW, et al. Feasibility of controlling COVID-19 outbreaks by isolation of cases and contacts. Lancet Glob Health 2020;8;e488-e96.

[14] Fu Y, Cheng Y, Wu Y. Understanding SARS-CoV-2-mediated inflammatory responses: from mechanisms to potential therapeutic tools. Virol Sin 2020;35;266-71.

[15] Rothan HA, Byrareddy SN. The epidemiology and pathogenesis of coronavirus disease (COVID-19) outbreak. J Autoimmun $2020 ; 109 ; 102433$. 